\title{
Ultrapermeable Composite Membranes Enhanced via Doping with Amorphous MOF Nanosheets
}

\author{
Min Liu, ${ }^{[\mathrm{a}]}$ Ke Xie, ${ }^{[\mathrm{a}]}$ Mitchell D. Nothling, ${ }^{[\mathrm{a}]}$ Lianhai Zu, ${ }^{[\mathrm{a}]}$ Shenlong Zhao, ${ }^{[\mathrm{b}]}$ Dalton J.E. Harvie, ${ }^{*[\mathrm{a}]}$ Qi- \\ ang $\mathrm{Fu},{ }^{*[\mathrm{c}]}$ Paul A. Webley, ${ }^{*[\mathrm{a}, \mathrm{d}]}$ and Greg G. Qiao*[a] \\ ${ }^{[a]}$ Department of Chemical Engineering, The University of Melbourne, Parkville, VIC 3010, Australia \\ ${ }^{[b]}$ School of Chemical and Biomolecular Engineering, The University of Sydney, Sydney, NSW, 2006 Australia \\ ${ }^{[c]}$ School of Civil and Environmental Engineering, University of Technology Sydney, NSW 2007 Australia \\ ${ }^{[d]}$ Department of Chemical Engineering, Faculty of Engineering, Monash University, Clayton, VIC 3800, Australia
}

\begin{abstract}
Thin film composite (TFC) polymeric membranes have attracted increasing interest to meet the demands of industrial gas separation. However, the development of high performance TFC membranes within their current configuration faces two key challenges: (i) the thickness-dependent gas permeability of polymeric materials (mainly polydimethylsiloxane (PDMS)) and (ii) the geometric restriction effect due to the limited pore accessibility of the underlying porous substrate. Here we demonstrate that the incorporation of trace $(\sim 1.8 \mathrm{wt} \%)$ amounts of amorphous metal-organic framework (MOF) nanosheets into the gutter layer of TFC assemblies can simultaneously address these two limitations by the creation of rapid, transmembrane gas diffusion pathways. Leveraging this strategy, we successfully fabricate a novel TFC membrane, consisting of a PDMS\&MOF gutter and an ultrathin $(\sim 54 \mathrm{~nm})$ poly(ethylene glycol) top selective layer via surface-initiated atom transfer radical polymerization (ATRP). The complete TFC membrane exhibits excellent processability and remarkable $\mathrm{CO}_{2} / \mathrm{N}_{2}$ separation permeance $\left(1,990 \mathrm{GPU}\right.$ with a $\mathrm{CO}_{2} / \mathrm{N}_{2}$ ideal selectivity of 39). This study reveals a strategy for the design and fabrication of a new TFC membrane system with unprecedented gas separation performance.
\end{abstract}

\section{INTRODUCTION}

Thin film composite (TFC) membranes for gas separation, usually consisting of a porous bottom support layer, a highly permeable intermediate gutter layer and an ultrathin top selective layer, are promising candidates to meet the requirements for high-throughput post-combustion $\mathrm{CO}_{2}$ capture. ${ }^{1-2} \mathrm{~A}$ polymeric intermediate gutter layer is essential within a TFC configuration, acting as a multi-functional coating, which can improve the compatibility between the top selective layer and the lower porous support, i.e. preventing the penetration of dilute polymer solution into the porous structure and rendering a smooth surface for coating a top selective layer. ${ }^{3}$ Frequently, the gutter layer is composed of polydimethylsiloxane (PDMS), which has been the most widespread choice in industry due to its relatively good chemical and thermal stability, as well as high gas permeability and excellent processability. ${ }^{4}$ However, the observed permeability of PDMS gutter layers documented in the literature deviates from the theoretical value as the thickness of the PDMS layer is reduced, though the permeability should be independent of film thickness. ${ }^{5}$ As an illustrative example, the observed $\mathrm{CO}_{2}$ permeability across a $\sim 230 \mathrm{~nm}$ PDMS layer on a microporous polyacrylonitrile (PAN) support is only 660 Barrer, much lower than the intrinsic permeability of PDMS (ca. 3,800 Barrer) at $35^{\circ} \mathrm{C} .{ }^{4,6}$ This discrepancy is suggested to be due to various non-equilibrium sorption-desorption effects dominating at the polymer interface, as well as morphological changes in the crosslinked polymer network. ${ }^{5,8}$ Furthermore, the pore infiltration and the geometric restriction in a
TFC membrane assembly resulting from the limited pore accessibility of porous substrates further exacerbate such thicknessdependent permeability. ${ }^{9-11}$ Consequently, these two issues handicap the development of high-performance TFC membranes.

In an effort to address these issues associated with gutter layers, the Park group reported an alternative Teflon (AF2400) based gutter layer, which displayed excellent $\mathrm{CO}_{2}$ permeance (> $30000 \mathrm{GPU}){ }^{8}$ More recently, our group has developed a gutter layer composed of microporous metal-organic framework (MOF) with similar gas permeance. ${ }^{12-13}$ However, the expensive Teflon and metal-organic framework (MOF) based gutter layers have relatively lower flexibility and processability. Furthermore, similar to other highly permeable polymers such as poly(1-(trimethylsilyl)-1-propyne) (PTMSP) and polymers of intrinsic microporosity (PIM),${ }^{14}$ amorphous Teflon also suffers from a physical aging effect, limiting its impact in an industrial setting. ${ }^{15}$ The key question here is: Can we enhance the performance of a PDMS gutter layer to the levels achieved for MOF, Teflon-, or PTMSP-based materials, while simultaneously offsetting the traditional thickness-dependent permeability and geometric restriction of porous substrates, without affecting their high processability?

Herein, we report a straightforward strategy to dramatically increase the gas permeance of conventional PDMS gutter layers by incorporating trace amounts of novel, ultrathin amorphous MOF nanosheets. We demonstrate that incorporating just $\sim 1.8$ $\mathrm{wt} \%$ amorphous MOF nanosheets into a $c a .230 \mathrm{~nm}$ PDMS gutter layer can result in over 3 -fold increase in gas permeance 
compared to pristine PDMS of similar thickness without compromising processability. Investigation via a combined experimental and computational approach reveals a unique mechanism behind the enhancement delivered by amorphous MOF nanosheets through limiting pore infiltration and providing accelerated transmembrane gas transport pathways. Such a straightforward doping method is readily generalized to a range of existing TFC materials, providing a powerful new strategy to directly improve the gas separation performance of current TFC membrane assemblies.

\section{RESULTS AND DISCUSSION}

Amorphous MOF Nanosheet Synthesis. MOFs are porous hybrid materials synthesized via linking inorganic metallic nodes and organic ligands through strong coordination bonds in a continuous manner. ${ }^{16-17}$ Most reported MOFs have clear crystalline X-ray diffraction (XRD) patterns as a result of their long-range coordination profiles. However, according to the MOF definition by the International Union of Pure and Applied Chemistry (IUPAC), ${ }^{18}$ there is no specific requirement for long-range order or crystallinity in a MOF structure. As a counterpoint, amorphous MOFs possess the basic constituents and connectivity of crystalline MOFs and are emerging as functional, porous materials for applications in reversible gas storage and controlled drug delivery. ${ }^{19}$ As suggested by their name, amorphous MOFs usually display broad, diffuse XRD patterns due to their lack of long-range periodic order caused by the aperiodic arrangements of their constituent atoms. In this work, ultrathin amorphous MOF nanosheets were firstly synthesized via a simple coordination modulation method. The ligand 2,5-dioxido-1,4benzenedicarboxylic acid (DOBDC) contains two types of metal-coordinating groups, namely: (i) the two carboxylic acids (-COOH) and (ii) the two hydroxy groups $(-\mathrm{OH})$. As illustrated in Figure 1a, the $\mathrm{Cu}^{2+}$ ions can coordinate with a capping molecule (acetonitrile $\left(\mathrm{CH}_{3} \mathrm{CN}\right)$ as a neutral Lewis base) and DOBDC simultaneously during the synthesis process. In this case, the interaction between metal and $\mathrm{CH}_{3} \mathrm{CN}$ as a neutral Lewis base is favored over the interaction between the metal and functional groups (- $\mathrm{COO}^{-}$and $-\mathrm{O}^{-}$) of the charged ligand. ${ }^{20}$ Due to the single coordination site of $\mathrm{CH}_{3} \mathrm{CN}$, the framework extension and crystal growth of the MOFs is distorted, resulting in an aperiodic arrangement of the building blocks and anisotropic growth of the MOFs into a two-dimensional (2D) amorphous structure (Figure 1a and Figure $\mathrm{S} 1$ ). In contrast, the preparation of crystalline analogue (MOF-74-Cu) via a conventional growth method results in rod-like particles (Figure S2).

The prepared amorphous MOF nanosheets appear disc-like in shape with a diameter of $\sim 2 \mu \mathrm{m}$ (Figure $1 \mathrm{~b}$ and c, Figure S3). The thickness of the nanosheets is around $6 \mathrm{~nm}$, as determined by atomic force microscopy (AFM, Figure 1b). In addition, no electron reflection was observed when assessing the nanosheets by high resolution transmission electron microscopy (TEM), confirming the amorphous nature of the prepared MOF nanosheets (Figure 1d). The X-ray diffraction (XRD) pattern of the amorphous MOF nanosheets reveals broad, diffuse peaks in comparison with crystalline MOF-74-Cu, ${ }^{20-23}$ offering further support for their amorphous structure (Figure 1e). Significantly, the amorphous MOF nanosheets display a similar attenuated total reflectance Fourier-transform infrared (ATR-FTIR) spectroscopy as the crystalline MOF-74-Cu (Figure S4), suggesting a similar coordination profile between the two morphologies. Specifically, both MOFs display decreased $\mathrm{O}-\mathrm{H}$ bending and stretching vibration intensities at 1300-1500 $\mathrm{cm}^{-1}$ and $2400-3400 \mathrm{~cm}^{-1}$, compared with the pristine DOBDC ligand. This can be attributed to the deprotonation and coordination with $\mathrm{Cu}^{2+}$ of the hydroxyl and carboxylate groups of the DOBDC ligand. In addition, the two MOFs showed a weak band at $1450-1500 \mathrm{~cm}^{-1}$ and a strong band at $1580-1600 \mathrm{~cm}^{-1}$ (red region), indicating the $\mathrm{C}-\mathrm{C}=\mathrm{C}$ asymmetric and symmetric stretching vibrations of the aromatic ring within the MOFs, respectively. The coordination nature of the amorphous MOF nanosheets was further investigated by an ex situ X-ray absorption spectroscopy (XAS). The resultant extended X-ray absorption fine structure (EXAFS) profile of the amorphous MOF nanosheets displayed a similar $\mathrm{Cu}-\mathrm{O}$ bonding to crystalline MOF-74- $\mathrm{Cu}$ at the main peak of $1.44 \AA$, along with decrease in peak intensities (Figure 1f). In addition, significant shifts were observed in the $\mathrm{Cu}-\mathrm{Cu}$ and $\mathrm{Cu}-$ $\mathrm{C}$ peaks between amorphous MOF nanosheets and crystalline MOF74-Cu (Figure 1f). Such decrease and movements can be chiefly attributed to the partial lack of DOBDC ligands around $\mathrm{Cu}$ atoms, leading to a relatively larger space for random coordination and shorted adjacent atom distances. This is confirmed by the X-ray absorption nearedge structure (XANES) peak intensity changes of the amorphous MOF nanosheets, in which decreased and increased peak intensities were observed at $c a .8995 \mathrm{eV}$ and at $c a .8986 \mathrm{eV}$, indicating the loss of axial ligands for $\mathrm{Cu}$ cations (Figure $\mathrm{S} 5)^{22,24}$
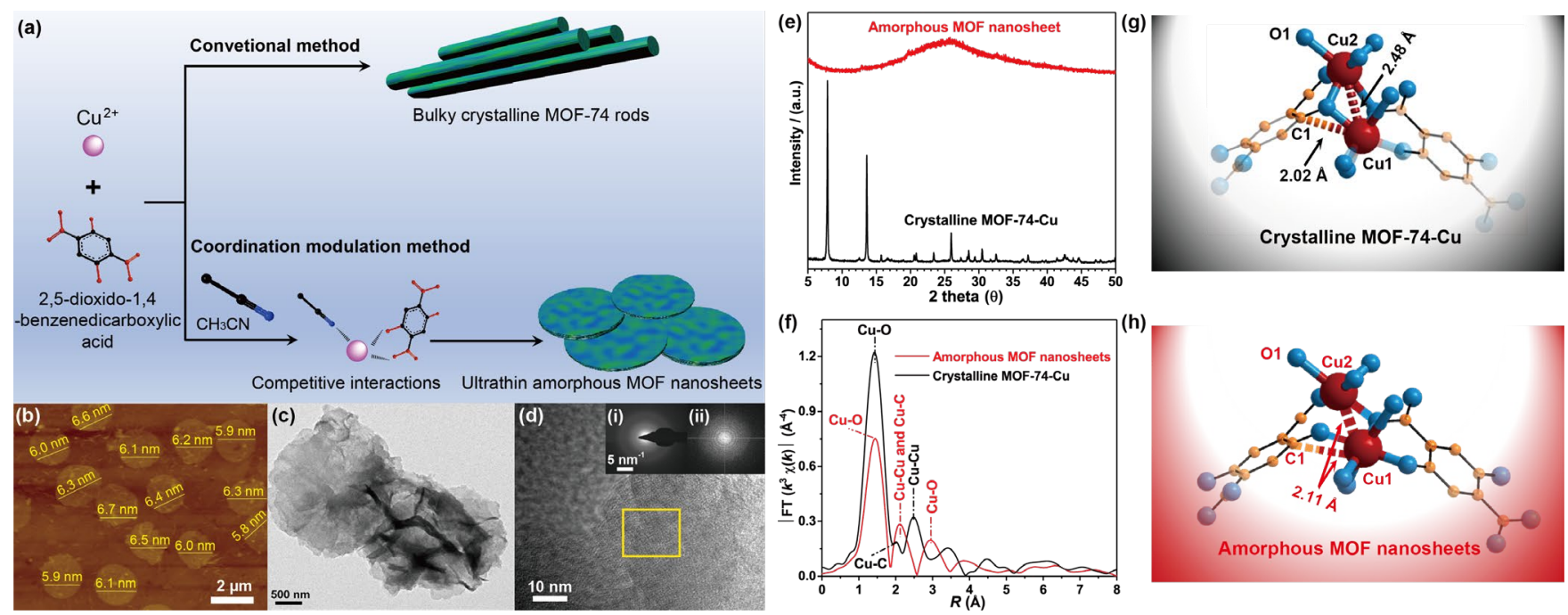

Figure 1. (a) Synthesis of ultrathin amorphous MOF nanosheets by a coordination modulation method. (b) AFM and (c) TEM images of the amorphous MOF nanosheets. The thickness of the amorphous MOF nanosheets is around $6 \mathrm{~nm}$ measured by AFM (indicated in yellow in (b)). (d) High resolution TEM image of the amorphous MOF nanosheets. Insets: (i) and (ii) illustrate the selected area electron diffraction (SAED) and fast Fourier transform (FFT) patterns of the yellow marked area, respectively. (e) XRD and (f) EXAFS spectra of the amorphous MOF nanosheets and crystalline MOF-74-Cu. ( $\mathrm{g}$ and $\mathrm{h}$ ) Proposed chemical bond structures of $(\mathrm{g})$ the crystalline MOF-74-Cu and (h) amorphous MOF nanosheets derived from EXAFS spectra. 
Examining the amorphous MOF nanosheets and crystalline MOF74-Cu using X-ray photoelectron spectroscopy (XPS) (Figure S6), revealed similar coordination properties between the two morphologies, with both exhibiting characteristic $\mathrm{Cu}(\mathrm{II}) 2 \mathrm{p}\left(\mathrm{Cu} 2 \mathrm{p}_{1 / 2}: 954.8 \mathrm{eV}, \mathrm{Cu}\right.$ 2p $\mathrm{p}_{3 / 2}: 934.9 \mathrm{eV}$ ), C 1s (C-C: $285.1 \mathrm{eV}, \mathrm{C}-\mathrm{O}: 287.4 \mathrm{eV}, \mathrm{O}-\mathrm{C}=\mathrm{O}: 289.1$ $\mathrm{eV})$ and $\mathrm{O} 1 \mathrm{~s}(\mathrm{C}-\mathrm{O}-\mathrm{Cu}: 532.2 \mathrm{eV}$ and $\mathrm{C}=\mathrm{O}: 533.7 \mathrm{eV})$ spectra. Such observations are consistent with a previous study by Dietzel et al..$^{25}$ where it was suggested that in the presence of an insufficiently strong base, only the two - $\mathrm{COOH}$ groups were deprotonated and coordinated with metal ions to form CPO-26 MOFs with a PtS type structure. The -OH groups participate indirectly by forming intramolecular hydrogen bonds with the oxygen atoms of the adjacent $-\mathrm{COO}^{-}$groups. To further confirm the coordination motif in the obtained amorphous MOF nanosheets, the DOBDC was firstly dissolved in an equimolar mixture of dimethylformamide- $d_{7}\left(\mathrm{DMF}-d_{7}\right)$ and acetonitrile- $d_{3}\left(\mathrm{CD}_{3} \mathrm{CN}-d_{3}\right)$ to mimic the experimental deprotonation conditions, resulting in an acidic solution with a $\mathrm{pH}$ of 5 6. As shown in Figure S7, only the peak representing the $\mathrm{Ar}-\mathrm{H}$ is observed in the ${ }^{1} \mathrm{H}$ NMR spectrum of the mixed solution. This result suggests both the $-\mathrm{COOH}$ and the $-\mathrm{OH}$ groups were in their deprotonated form, and available for coordination with $\mathrm{Cu}^{2+}$ during the MOF synthetic process. ${ }^{26-27}$

Significantly, both the amorphous MOF nanosheets and their crystalline MOF-74-Cu equivalent exhibit type II $\mathrm{N}_{2}$ adsorption/desorption isotherms at $77 \mathrm{~K}$, indicating similar structural profiles as well as strong interactions between MOFs and $\mathrm{N}_{2}$ (Figure S8 and S9). The amorphous MOF nanosheets show relatively lower surface area $\left(97.9 \mathrm{~m}^{2} / \mathrm{g} v s\right.$. $\left.580.7 \mathrm{~m}^{2} / \mathrm{g}\right)$ and total pore volume $\left(0.02 \mathrm{~cm}^{3} / \mathrm{g}\right.$ vs. $\left.0.69 \mathrm{~cm}^{3} / \mathrm{g}\right)$ than those of MOF-74-Cu as expected. This can be attributed to (i) the amorphous nature of the amorphous MOF nanosheets and (ii) the severe aggregation of nanosheets after physical drying, ${ }^{13}$ which cannot present the porous structure of an ultrathin nanosheet observed by TEM (Figure 1c) and SEM (Figure S1). Importantly, the amorphous MOF nanosheets displayed a higher $\mathrm{CO}_{2}$ adsorption than $\mathrm{N}_{2}$ at $373 \mathrm{~K}$ due to the stronger interactions between $\mathrm{CO}_{2}$ and $\mathrm{Cu}^{2+}$ open metal sites (Figure S10). Examining the amorphous MOF nanosheets by thermogravimetric analysis (TGA) reveals a lower thermal stability in comparison with the crystalline MOF-74-Cu, due to their amorphous character (Figure S11). Interestingly, only the copper-based MOFs displayed a 2D morphology, while other MOFs composed of alternative metal nodes (Mg, Al, Fe, Co, Ni, Zn, Mn, Zr) via the same method exhibited different morphologies (Figure S12). We attribute this difference to the varied coordination profiles of the different metal ions. ${ }^{28}$

Membrane Preparation and Properties. Following successful preparation of the amorphous MOF nanosheets, we next sought to examine their impact on the gas permeation when doped into a PDMS gutter layer. Briefly, the amorphous MOF nanosheets were mixed with amino-terminated PDMS, which were covalently linked by 1,3,5-benzenetricarbonyl trichloride. This was followed by spin-coating of the reacted formulation onto pre-wetted PAN substrates to produce PDMS\&MOF gutter layers (Figure 2a). No detectable changes to the infrared spectra were observed between PDMS\&MOF and pure PDMS (Figure S13), failing to account for the introduction of amorphous MOF nanosheets. This is attributed to the limited number of specific nanosheet surface contacts compared to the bulk PDMS phase. ${ }^{29}$ Notably, the resultant gutter layer exhibited low roughness, similar to a pristine PDMS example, indicating an even dispersion of amorphous MOF nanosheets within the PDMS matrix (Figure S13a and b). It is known that many MOFs are not stable in highly acidic solution, as indicated by the acid-base theory (i.e. strong acids will replace weak acids). However, in the current reaction, the generated $\mathrm{HCl}$ molecules have very low solubility in the employed hydrophobic hexane, limiting their interaction with the amorphous MOF nanosheets. In addition, we also observed some amorphous MOF aggregations after spin-coating via AFM measurements (Figure S13c). This result further demonstrates that the amorphous MOF nanosheets are chemically stable during the spin-coating process. (a)

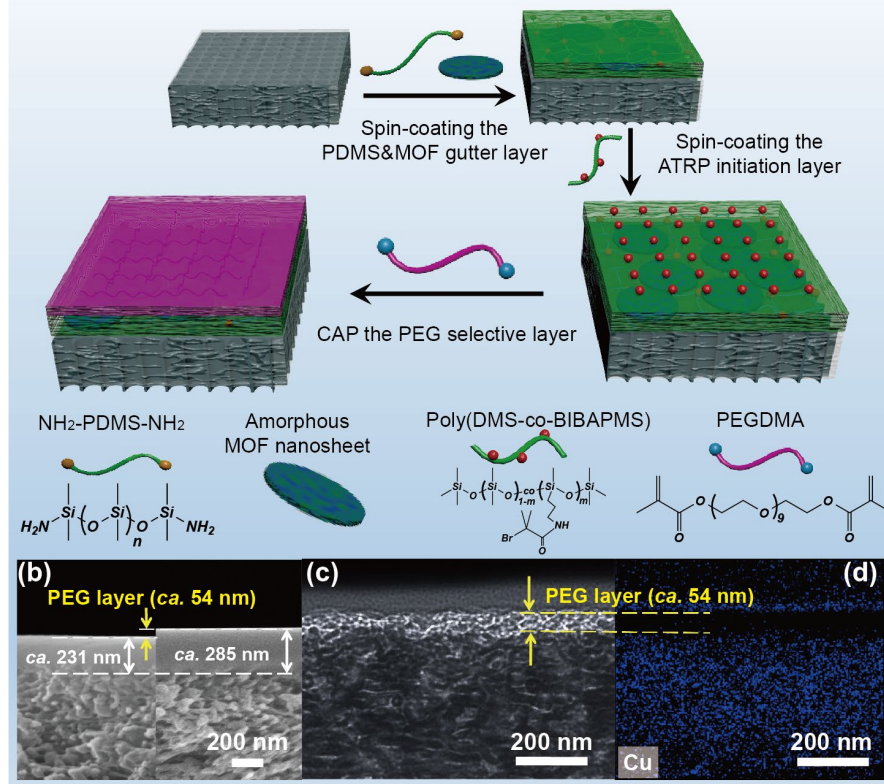

(e): M1

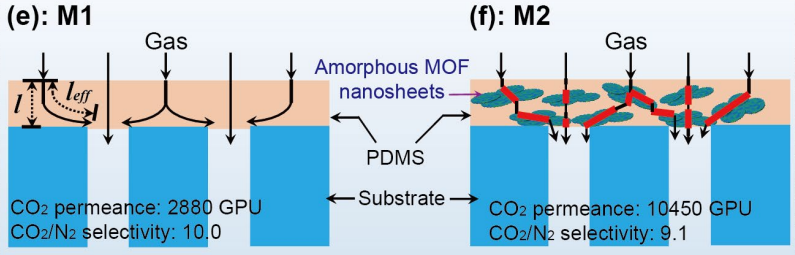

(g): M3
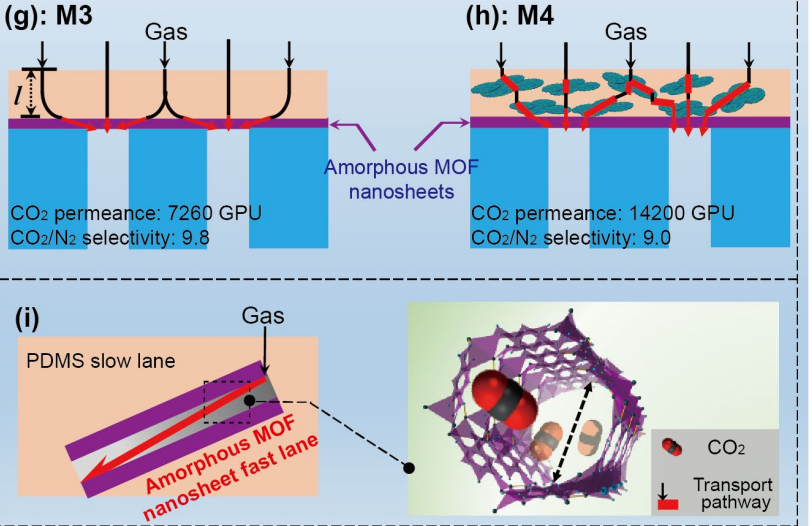

Figure 2. (a) Schematic illustration of the preparation of the TFC membranes by continuous assembly of a polymer (CAP) technology. (b) Crosssectional SEM image of the TFC membrane: (left) PDMS\&MOF initiator layer and (right) the final TFC membrane. (c) Cross-sectional TEM image of the TFC membrane and (d) the corresponding Cu K energy-dispersive X-ray spectroscopy (EDX) image. The yellow line marked areas in (c) and (d) represent the ultrathin PEG selective layer and the blue color in (d) indicates the presence of amorphous MOF nanosheets in PDMS. (e: M1) Illustration of the geometric restriction effect on the gas diffusion length. A membrane with a gutter layer thickness of $l$ will have an effective diffusion length $\left(l_{e f f}\right)$ larger than $l$ due to the limited porous accessibility of the substrate. (f-h) Illustration of enhancing gas permeation rate via (f: M2) embedding ultrathin amorphous MOF nanosheets into PDMS matrix, (g: M3) introducing a porous amorphous MOF nanosheet layer between PDMS gutter layer and substrate, and (h: M4) inserting a porous amorphous nanosheet layer between PDMS\&MOF gutter layer and substrate. The amorphous MOF nanosheet layer was coated onto PAN substrate by vacuum filtration ( $\mathrm{CO}_{2}$ permeance: $38000 \mathrm{GPU}$ and $\mathrm{N}_{2}$ permeance: $\left.40000 \mathrm{GPU}\right)$, followed by spin-coating a PDMS or PDMS\&MOF layer $(2.0 \mathrm{w} / \mathrm{v} \%, 1000 \mathrm{rpm}, 10 \mathrm{~s})$. (i) Illustration of ultrafast gas diffusion accelerated by the amorphous MOF nanosheets in PDMS matrix. 
Unexpectedly, the prepared gutter layer with only $\sim 1.8 \mathrm{wt} \%$ amorphous MOF inclusion exhibited a more than 3-fold increase in $\mathrm{CO}_{2}$ permeance (10450 GPU at 1.0 bar, Figure 2f: M2) with a good $\mathrm{CO}_{2} / \mathrm{N}_{2}$ ideal selectivity of 9.1 in comparison with pristine PDMS gutter layers $\left(2,880 \mathrm{GPU}, \mathrm{CO}_{2} / \mathrm{N}_{2}=10\right.$, Figure 2e: M1) of similar thickness. Under further investigation, we found that the enhanced permeance can be attributed to three possible factors: Firstly, XRD characterization reveals that the embedded amorphous MOF nanosheets can increase the disorder degree of PDMS chain packings (i.e. lowering the local density of PDMS) and thereby increase the free volume within the PDMS layer, favoring an increase in diffusion properties of the formed membrane (Figure S15). ${ }^{30}$ Secondly, the embedded amorphous MOF nanosheets may mitigate the pore infiltration of PDMS, reducing actual gas transport length. Thirdly, the hierarchical porous structure of the amorphous MOF nanosheets provides a fast diffusion pathway, which can significantly reduce the geometric restriction effect of the composite configuration (Figure 2i).$^{9,31}$

In order to verify the second and third hypothesis, we conducted two control experiments. Firstly, we coated an ultrathin amorphous MOF nanosheet layer on a PAN substrate via vacuum filtration with a similar mass to that employed in the previous experiment. Then a PDMS or PDMS\&MOF layer was spin-coated on top of the amorphous MOF layer (Figure 2, g: M3 and h: M4). In the case of inserting an amorphous MOF nanosheet layer between the PDMS and substrate (Figure 2g: M3), the resultant membrane showed a $\mathrm{CO}_{2}$ permeance of 7260 GPU together with a $\mathrm{CO}_{2} / \mathrm{N}_{2}$ ideal selectivity of 9.8. The increase in $\mathrm{CO}_{2}$ permeance indicates that the geometric restriction of the porous substrate for $\mathrm{CO}_{2}$ permeance has been essentially mitigated (or removed) by the fast gas diffusion through the porous amorphous MOF nanosheet layer, in comparison with pristine PDMS gutter layer (Figure 2e: M1). Further replacing the pristine PDMS layer with a PDMS\&MOF layer, the $\mathrm{CO}_{2}$ permeance reached 14220 GPU along with a $\mathrm{CO}_{2} / \mathrm{N}_{2}$ ideal selectivity of 9.0 (Figure $2 \mathrm{~h}$ : M4). When comparing the separation performance illustrated in Figure 4, the embedding of amorphous MOF nanosheets into PDMS matrix could substantially enhance gas separation performance, indicating both the substrate geometric restriction and the thickness-dependent gas permeability issues have been substantially resolved.

To further probe these experimental results, a complementary quantitative diffusion simulation study was conducted to probe how the amorphous MOF nanosheets are increasing the membrane permeability. ${ }^{32}$ The simulations (detailed further in METHODS) consider the axisymmetric diffusion of $\mathrm{CO}_{2}$ around a prototypical substrate pore, and model the permeability of the PDMS\&MOF material using a composite resistance-based model that accounts for the aspect ratio of the MOF inclusions. Hence, in the model the (tensor) permeability of the PDMS\&MOF material is different in the horizontal (radial) and vertical (axial) directions, capturing in the diffusion modelling some of the geometrical aspects of the included amorphous MOF nanosheet structures. Simulations were conducted both with and without amorphous MOF nanosheet boundary layers.

First, we characterized the pristine substrate and a surface porosity of $\sim 3.5 \%$ along with an average pore size of $\sim 11.5 \mathrm{~nm}$ was obtained (Figure S16 and S17). As PDMS suffers from thickness-dependent gas permeability and pore infiltration, we first simulated the $\mathrm{CO}_{2}$ permeation of the PAN/MOF/PDMS membrane (Figure $2 \mathrm{~g}$ : M3). This is because the inserted amorphous MOF nanosheet layer can not only function as fast diffusion lanes to remove the geometric restriction, but also prevent the penetration of PDMS into the lower PAN substrate. As a result, the simulated $\mathrm{CO}_{2}$ permeability of PDMS in the PAN/MOF/PDMS membrane should be the "real" performance of a $230 \mathrm{~nm}$ "free standing" PDMS membrane, which can be further used to simulate the other three membranes. In the first simulation study, the $\mathrm{CO}_{2}$ permeance of $7300 \mathrm{GPU}$ for the PAN/MOF/PDMS membrane (Figure 2g: M3) was used to determine the $\mathrm{CO}_{2}$ permeability of the bulk PDMS material. Iteration showed that a permeability of 1660 Barrer for bulk PDMS produced the correct $\mathbf{M} 3$ membrane performance, and was hence used for the bulk PDMS permeability for the other three membrane configurations. For the PAN/MOF/PDMS\&MOF (Figure 2h: M4) membrane, it was found that the simulated $\mathrm{CO}_{2}$ permeance was the same as the tested performance (14200 GPU) when the aspect ratio of the amorphous MOF inclusions was set at $\sim 5.9$ (Figure $3 \mathrm{~b}$ ). The simulated high aspect ratio of the amorphous MOF nanosheets can be attributed to the finite width of the amorphous MOF nanosheets and that they cannot be perfectly flat within the thin PDMS matrix, leading to the aggregation or self-folding of amorphous MOF nanosheets in PDMS.
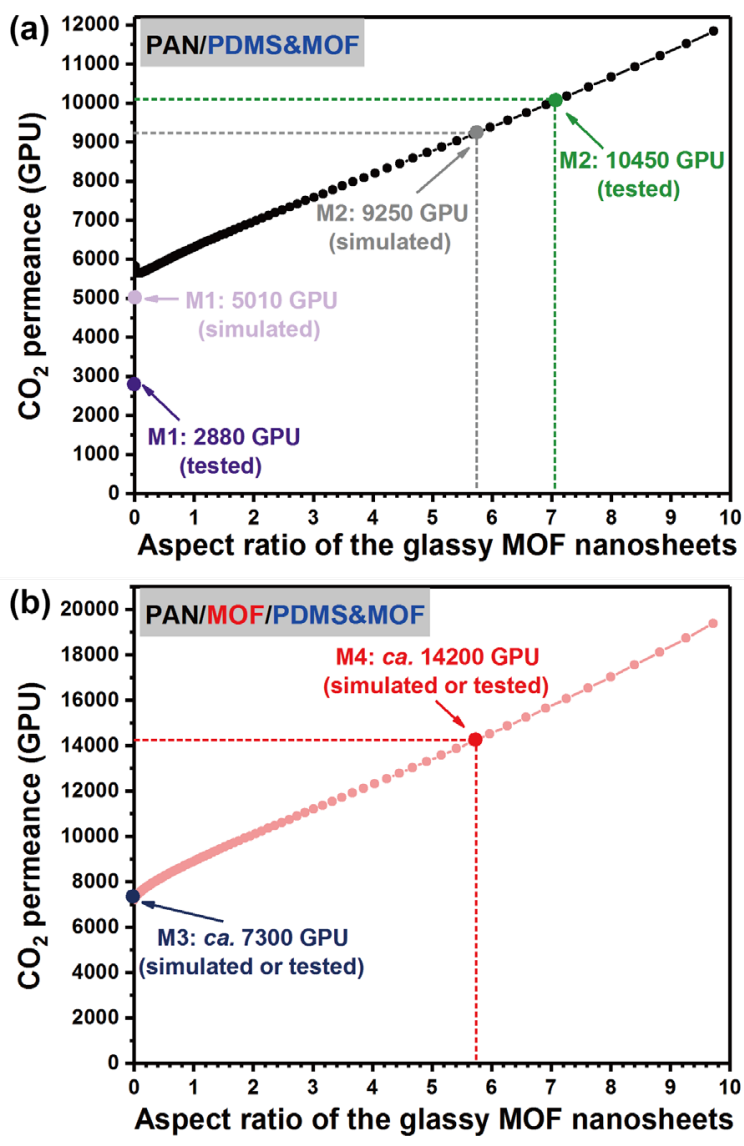

Figure 3. Comparison of tested and simulated $\mathrm{CO}_{2}$ permeance of (a) M1: PAN/PDMS (with $0 \mathrm{v} / \mathrm{v} \%$ amorphous MOF nanosheets loading) and M2: PAN/PDMS\&MOF, and (b) M3: PAN/MOF/PDMS (with 0 $\mathrm{v} / \mathrm{v} \%$ amorphous MOF nanosheets loading) and M4: PAN/MOF/PDMS\&MOF membranes with different amorphous MOF nanosheet aspect ratios in PDMS.

With the diffusion model calibrated using the two \&MOF membrane configurations (M3 and M4), the permeance of the remaining two non\&MOF membrane configurations (M1 and M2) was interpreted using the model. Simulating the performance of the PAN/PDMS\&MOF membrane (Figure 2f: M2), we found the $\mathbf{M} 2$ displayed a $\mathrm{CO}_{2}$ permeance of $9250 \mathrm{GPU}$ at the same amorphous MOF nanosheet inclusion aspect ratio of 5.9 , which is broadly consistent $(\sim 11.5 \%$ lower) with the measured value (9250 GPU vs. 10450 GPU, Figure 3a). This correspondence suggests that the model is capturing the different diffusion effects caused by the amorphous MOF nanosheet inclusions and amorphous MOF nanosheet layers. The small difference between permeance results for $\mathbf{M 2}$ may be attributed to the variation in the self-folding behavior of the amorphous MOF nanosheets, or the morphological changes in the crosslinked polymer network around the amorphous MOF nanosheet inclusions.

While the above three simulated $\mathrm{CO}_{2}$ permeance results were close to the tested values using a PDMS $\mathrm{CO}_{2}$ permeability of 1660 Barrer (and amorphous MOF nanosheet inclusion aspect ratio of $\sim 5.9$ ), the simulated $\mathrm{CO}_{2}$ permeance of the PAN/PDMS (M1) membrane is much higher than the simulated data (5010 GPU vs. 2880 GPU). This can be attributed to the penetration of PDMS into the PAN substrate during membrane fabrication process, leading to an effectively thicker PDMS 
gutter layer than that measured value by SEM. To quantify the penetration, a further calculation on the pore infiltration length was conducted by considering the resistance of infiltration (Equation 1, detailed further in the supplementary information):

$$
\frac{1}{Q_{t}}=\frac{1}{Q_{m}}+\frac{l_{p}}{\emptyset \times P_{m}}
$$

where $Q_{t}$ and $Q_{m}$ are the tested and simulated $\mathrm{CO}_{2}$ permeance of the PAN/PDMS membrane (M1), respectively; $\emptyset$ is the porosity of the
PAN substrate; $P_{m}$ is the $\mathrm{CO}_{2}$ permeability of PDMS (1660 Barrer) According to the calculation, the penetration length is around $8.5 \mathrm{~nm}$, which is small compared to the overall thickness of the PDMS layer, but contributes significantly to the overall diffusion resistance due to the relatively low porosity of the PAN substrate. Indeed, such a minor penetration caused $42.5 \%$ loss of $\mathrm{CO}_{2}$ permeance, highlighting the importance of removing the geometric restriction generated from the lower substrate.
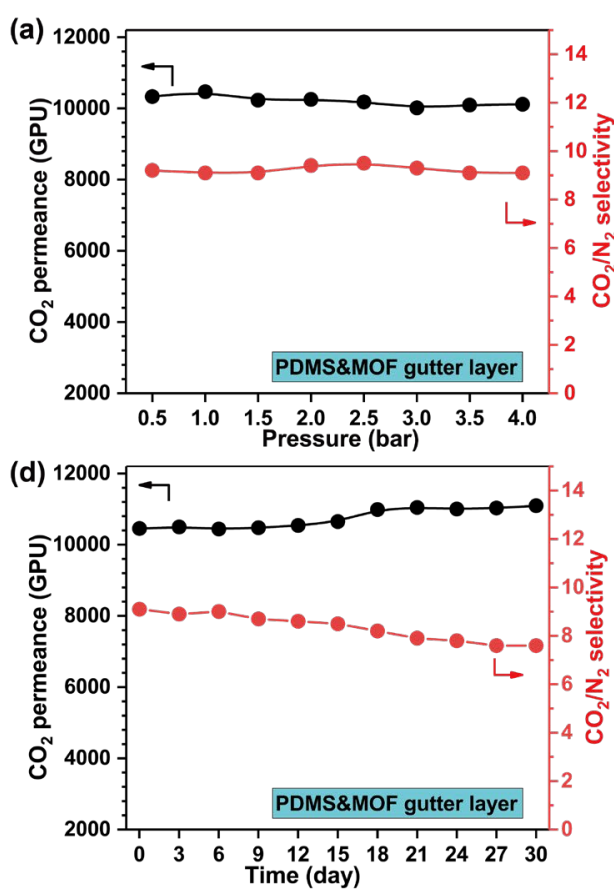

(b)

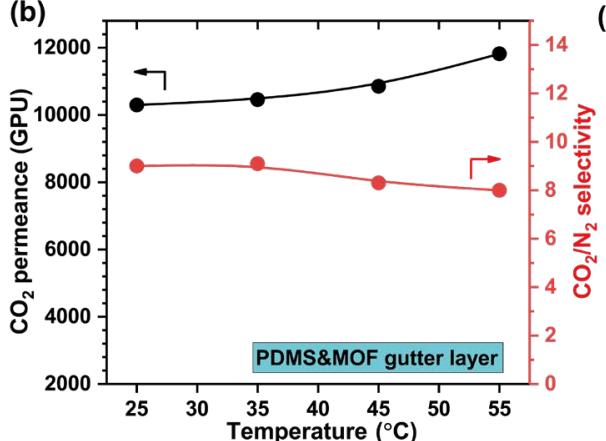

(e)

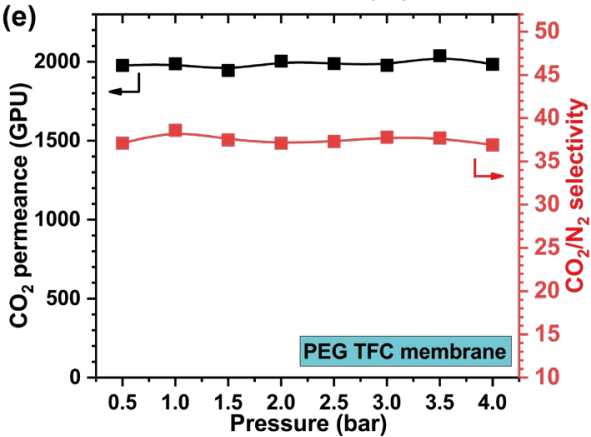

(c)
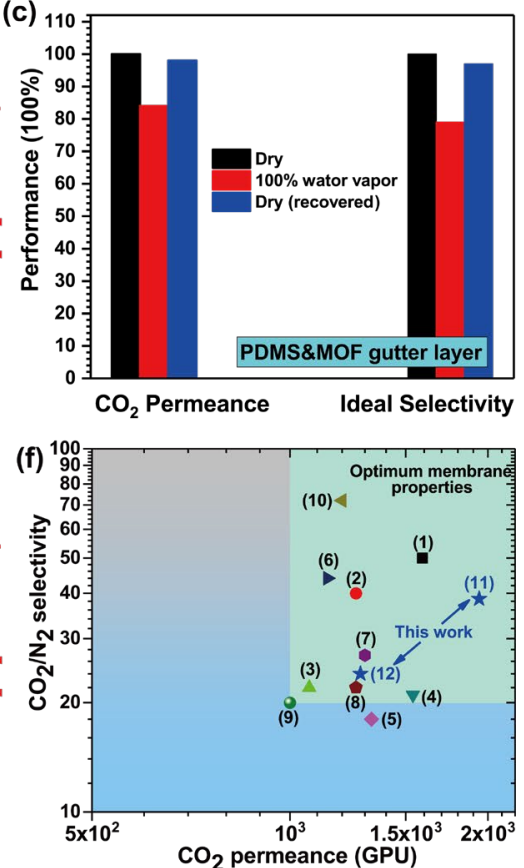

Figure 4. The $\mathrm{CO}_{2} / \mathrm{N}_{2}$ separation performance of PDMS\&MOF gutter layer under variable conditions: (a) $0.5-4.0$ bar, (b) $25-55{ }^{\circ} \mathrm{C}$, (c) in the presence of $100 \%$ water vapor, and (d) long-term stability within 30 days. (e) The $\mathrm{CO}_{2} / \mathrm{N}_{2}$ separation performance of complete PEG-based TFC membranes under a pressure range of $0.5-4.0$ bar. (f) The $\mathrm{CO}_{2} / \mathrm{N}_{2}$ selectivity versus $\mathrm{CO}_{2}$ permeance plot comparing the performance of the prepared TFC membranes with other state-of-the-art TFC membranes reported in the literature. The region designated as the target performance area for postcombustion $\mathrm{CO}_{2}$ capture was inferred from Merkel et al. ${ }^{33}$ Further figure details are summarized in Table 1.

Table 1. Comparison of the $\mathrm{CO}_{2} / \mathrm{N}_{2}$ separation performance of film composite membranes using PDMS as gutter layers. The facilitated transport membranes were excluded.

\begin{tabular}{|c|c|c|c|c|c|c|}
\hline \multirow[b]{2}{*}{ Entry and Reference } & \multicolumn{3}{|c|}{ Membrane configuration } & \multirow[b]{2}{*}{$\begin{array}{l}\text { Operation } \\
\text { conditions }^{[b]}\end{array}$} & \multirow[b]{2}{*}{$\begin{array}{l}\mathrm{CO}_{2} \text { perme- } \\
\text { ance (GPU) }\end{array}$} & \multirow[b]{2}{*}{$\begin{array}{l}\mathrm{CO}_{2} / \mathrm{N}_{2} \\
\text { ideal se- } \\
\text { lectivity }\end{array}$} \\
\hline & Selective layer & Gutter layer & $\begin{array}{l}\text { Total } \\
\text { thickness } \\
(\mathrm{nm})^{[\mathrm{a}]}\end{array}$ & & & \\
\hline (1) Energy Environ. Sci. 2011, 4, 4656 & Polyactive & PDMS & 130 & $30^{\circ} \mathrm{C}$ & 1590 & 50 \\
\hline (2) Energy Environ. Sci. 2016, 9, 434 & PEG & PDMS & $>240$ & $35^{\circ} \mathrm{C}, 3.5$ bar & 1260 & 40 \\
\hline (3) J. Mater. Chem. A 2013, 1, 13769 & Pebax $^{\circledR} 2533 /$ HMA-PEO & PDMS & 600 & $35^{\circ} \mathrm{C}, 3.5 \mathrm{bar}$ & 1070 & 22 \\
\hline (4) Ind. Eng. Chem. Res. 2016, 55, 8364 & Pebax $^{\circledR} 1657 /$ P 1 & PDMS & 675 & $35^{\circ} \mathrm{C}, 3.5 \mathrm{bar}$ & 1538 & 21 \\
\hline (5) J. Membr. Sci. 2016, 499, 191 & $\operatorname{Pebax}^{\circledR} 2533 /$ P21-4.3 & PDMS & 910 & $35^{\circ} \mathrm{C}, 3.5 \mathrm{bar}$ & 1330 & 18 \\
\hline (6) Nanoscale 2016, 8, 8312 & PEG/FeDA & PDMS & 450 & $35^{\circ} \mathrm{C}, 3.4$ bar & 1140 & 44 \\
\hline (7) J. Mater. Chem. A 2015, 3, 14876 & Pebax $^{\circledR} 2533 /$ SNP1 & PDMS & 550 & $35^{\circ} \mathrm{C}, 3.4 \mathrm{bar}$ & 1000 & 20 \\
\hline (8) J. Membr. Sci. 2016, 515, 54 & $\mathrm{PEG} / \mathrm{PEI}-\mathrm{SiO}_{2}$ & PDMS & 350 & $35^{\circ} \mathrm{C}, 3.4 \mathrm{bar}$ & 1300 & 27 \\
\hline (9) J. Membr. Sci. 2017, 535, 350 & Polyactive/P\&MOF & PDMS & $>300$ & $35^{\circ} \mathrm{C}, 3.0$ bar & 1260 & 22 \\
\hline $\begin{array}{l}\text { (10) ACS Appl. Mater. Interfaces, 2020, } 12 \text {, } \\
33196\end{array}$ & Pebax $^{\circledR} 1657$ & PDMS & 410 & $25^{\circ} \mathrm{C}, 3.0 \mathrm{bar}$ & 3300 & 37 \\
\hline (11) This work & PEG & PDMS\&MOF & 285 & $35^{\circ} \mathrm{C}, 1.0$ bar & 1990 & 39 \\
\hline (12) This work* & PEG & PDMS\&MOF & 285 & $\begin{array}{l}35^{\circ} \mathrm{C}, 1.0 \text { bar } \\
\mathrm{CO}_{2} / \mathrm{N}_{2}=10 / 90^{*}\end{array}$ & $1280^{*}$ & $24^{*}$ \\
\hline
\end{tabular}

[a] The total thickness represents the thickness of selective layer and gutter layer. [b] The listed work are the results of single gas separation tests. The results of the mixed gas $\left(\mathrm{CO}_{2} / \mathrm{N}_{2}=10 / 90\right)$ separation tests of this work have been marked with *.

We also investigated the type of MOFs and the morphological influence of MOF fillers on gas permeance through PDMS membranes. Of particular note, all of the examined MOFs are nanoparticles. As shown in Figure S18, MOF-Zr $(\sim 30 \mathrm{~nm}$, Figure S12g) and MOF-Al $(\sim 50 \mathrm{~nm}$,
Figure S12h) nanoparticles were poorly dispersible in hexane, resulting in a lower mass loading in the doped PDMS matrix compared to amorphous MOF nanosheets. The resultant PDMS\&MOF-Al and PDMS\&MOF-Zr membranes presented a $\mathrm{CO}_{2}$ permeance of 9700 
GPU and 12800 GPU together with a $\mathrm{CO}_{2} / \mathrm{N}_{2}$ selectivity of 4.7 and 2.5 , respectively. The improved $\mathrm{CO}_{2}$ permeance together with the decreased $\mathrm{CO}_{2} / \mathrm{N}_{2}$ selectivity are indicative of aggregation of the MOF nanoparticles, leading to the formation of defects (Figure S20). In addition, the observed MOF aggregations further indicate the generated $\mathrm{HCl}$ has a negligible influence on MOF stability during the PDMS crosslinking process. Considering the high gas separation performance along with high processability of the amorphous MOF nanosheets blended PDMS gutter layer, the fabricated membranes (Figure 2f: M2) were further tested under variable gas separation conditions. Significantly, the PMDS\&MOF gutter layer showed a constant $\mathrm{CO}_{2} / \mathrm{N}_{2}$ separation performance under a pressure between $0.5-4.0$ bar (Figure $4 \mathrm{a}$ ). However, an increasing trend of gas permeance along with loss of gas selectivity was observed under elevated temperatures. This result can be attributed to the well-known plasticization of PDMS chains at high temperatures (Figure 4b). The stability of the prepared PDMS\&MOF gutter layer in the presence of water vapor $(100 \%$ relative humidity) was also examined, revealing a $16 \%$ loss of $\mathrm{CO}_{2}$ permeance together with a $21 \%$ loss of $\mathrm{CO}_{2} / \mathrm{N}_{2}$ selectivity due to the competitive sorption effect of $\mathrm{H}_{2} \mathrm{O}$ (Figure 4c). Importantly, the PDMS\&MOF gutter layer recovers its original separation performance after being dried under vacuum, highlighting its high stability towards water vapor.

Interestingly, the PDMS\&MOF gutter layer displayed enhanced thermal stability compared to the pristine PDMS counterpart (Figure S21). This result may be attributed to the strong interaction between PDMS and the amorphous MOF nanosheets. Both the $\mathrm{CO}_{2}$ permeance and $\mathrm{CO}_{2} / \mathrm{N}_{2}$ ideal selectivity of the PDMS\&MOF gutter layer remained constant after storing in air for two weeks at $35^{\circ} \mathrm{C}$ (Figure 4d). However, upon further extending the testing time to three weeks, the $\mathrm{CO}_{2}$ permeance increased around $10 \%$ compared to the initial performance along with a $20 \%$ loss of $\mathrm{CO}_{2} / \mathrm{N}_{2}$ ideal selectivity. This result can be attributed to the accelerated $\mathrm{CO}_{2}$ induced plasticization effect in TFC membranes, ${ }^{34}$ whereby PDMS interchain interactions were disrupted under the solvating effect of polarizable $\mathrm{CO}_{2}$ molecules, leading to faster gas permeation and a loss of selectivity. The PDMS\&MOF membranes displayed good structural integrity and flexibility, maintaining their original shape and separation performance after being bent to a large degree for one week (Figure S22). Increasing the mass loading of the amorphous MOF nanosheets in the PDMS layer to $\sim 3.6 \mathrm{wt} \%$ resulted in increased roughness at the air-polymer interface (Figure $\mathrm{S} 13 \mathrm{~b})$, along with an increased $\mathrm{CO}_{2}$ permeance (12100 GPU) and an attenuated $\mathrm{CO}_{2} / \mathrm{N}_{2}$ ideal selectivity $\left(\mathrm{CO}_{2} / \mathrm{N}_{2}=7.1\right)$. This is attributed to the low dispersity and strong aggregation tendency of the amorphous MOF nanosheets in nonpolar solvents, leading to an exposed population of amorphous MOF nanosheets close to the surface. This phenomenon is exacerbated by the use of three-dimensional MOF nanoparticle as additives (Figure S18 and S19). Therefore, thoroughly dispersed porous nanosheets in a PDMS matrix are a desirable trait for the preparation of high-permeance PDMS gutter layers.

For economic post-combustion $\mathrm{CO}_{2}$ capture, a high $\mathrm{CO}_{2}$ permeance $(>1,000 \mathrm{GPU})$ along with good $\mathrm{CO}_{2} / \mathrm{N}_{2}$ selectivity $(>20)$ are desirable for membrane-based systems. ${ }^{33}$ In addition, process constraints, such as the low feed pressure ( $c a .1 \mathrm{bar}$ ), high volume, and low $\mathrm{CO}_{2}$ concentration $(15-16 \%)$ of flue gas as well as a high $\mathrm{CO}_{2}$ removal requirement (50-90\%) further challenge the development of viable membrane designs. ${ }^{35}$ Compression of flue gas to high pressures to assist membrane permeation should be avoided to conserve the energy cost of such unit processes. For example, $\sim 20 \%$ of the produced energy of power plants would be consumed when compressing the feed to a pressure of $5 \mathrm{bar}^{33}$ To demonstrate the potential of our approach in a complete TFC membrane assembly for post-combustion $\mathrm{CO}_{2}$ capture, an integrated TFC membrane was fabricated by growing a polymeric selective layer onto the crosslinked PDMS\&MOF gutter layer via a continuous assembly of polymer (CAP) nanotechnology (Figure 2a). The resultant composite membrane was then cut using a focused ion beam (FIB) and the cross-section was imaged using TEM to reveal the morphology of the individual layers (Figure 2, c and d). From this analysis, the thickness of the selective layer is determined to be $\sim 54 \mathrm{~nm}$, agreeing well with SEM measurements (Figure 2b).

In single gas separation tests, the resultant TFC membranes exhibited a $\mathrm{CO}_{2}$ permeance of $1,990 \mathrm{GPU}$ with a $\mathrm{CO}_{2} / \mathrm{N}_{2}$ ideal selectivity of
39 at $35{ }^{\circ} \mathrm{C}$ feed pressure of $1 \mathrm{bar}$, positioning this membrane well inside the target characteristics for post-combustion $\mathrm{CO}_{2}$ capture (Figure 4, e and f, Table 1). Significantly, the prepared TFC membrane maintains such separation performance under a broad feed pressure range between $0.5-4.0$ bar. To the best of our knowledge, this is the highest level of $\mathrm{CO}_{z}$-permeance yet reported for a flexible composite membrane employing a PDMS based gutter layer. Such dramatic increase in gas permeance is attributed to the decrease in gas permeation resistance of the gutter layer via the amorphous MOF nanosheets incorporation. ${ }^{13}$ Comparing this performance with TFC membranes incorporating a pristine PDMS gutter layer (1260 GPU, Entry 2, Table 1) ${ }^{6}$ reveals a $58 \%$ increase in $\mathrm{CO}_{2}$ permeance while maintaining a similar $\mathrm{CO}_{2} / \mathrm{N}_{2}$ selectivity, corresponding to a $\sim 30 \%$ decrease in capture cost ( $\sim$ US\$2 2 vs. $\sim$ US\$31, Figure S23). In addition, such a capture cost of this newly designed membrane is much lower than those of state-ofthe-art TFC membranes (Figure S23). To further validate this design, the fabricated TFC membranes were also examined in a mixed gas separation $\left(\mathrm{CO}_{2} / \mathrm{N}_{2}=10 / 90\right)$ scenario. Due to the lower $\mathrm{CO}_{2}$ concentration and diffusion, a lower $\mathrm{CO}_{2}$ permeance of $1280 \mathrm{GPU}$ and $\mathrm{CO}_{2} / \mathrm{N}_{2}$ permselectivity of 24 was observed, in line with expectations (Figure $6 \mathrm{f}$ and Entry 12, Table 1). Such performance maintains compliance with the economic requirements for post-combustion $\mathrm{CO}_{2}$ capture, highlighting the excellent potential of this technology for industrial TFC membrane development

\section{CONCLUSIONS}

In conclusion, we report a straightforward strategy to reduce the gas transport resistance of PDMS gutter layers by introducing trace amounts of ultrathin amorphous MOF nanosheets into a PDMS matrix. Our MOF-doping strategy mitigates the issues associated with conventional composite membranes, including the thickness-dependent gas permeability of PDMS materials and the geometric restrictions of porous substrates. The prepared PDMS\&MOF gutter layer displayed significantly enhanced gas permeance, which can be attributed to i) increases in the free fractional volume of the PDMS layer following inclusion of the amorphous MOF nanosheets, ii) decrease in pore infiltration by PDMS, and iii) the presence of fast gas transport lanes introduced by the embedded amorphous MOF nanosheets. By employing PDMS\&MOF as a gutter layer in a complete TFC assembly, the fabricated membranes exhibited $1,990 \mathrm{GPU}$ with a $\mathrm{CO}_{2} / \mathrm{N}_{2}$ ideal selectivity of 39 at $35^{\circ} \mathrm{C}$ feed pressure of 1 bar. This study thus reveals an avenue for the development of scalable, next generation TFC membranes with high-performance and high processability for industrial gas separation.

\section{METHODS}

Synthesis of MOFs. Bulk MOF-74-Cu was synthesized using the previously published procedures. ${ }^{20}$ The amorphous MOF nanosheets were synthesized by the coordination modulation method. Typically, DOBDC $(36.0 \mathrm{mg})$ and $30.0 \mathrm{mg}$ of $\mathrm{Cu}\left(\mathrm{NO}_{3}\right)_{2} \cdot 2.5 \mathrm{H}_{2} \mathrm{O}$ were dissolved in a glass vial containing a mixed solvent $(4.0 \mathrm{~mL}$ of DMF and $4.0 \mathrm{~mL}$ of $\mathrm{CH}_{3} \mathrm{CN}$ ). The glass vial was heated at $313 \mathrm{~K}$ for $24 \mathrm{~h}$ in static conditions. The reaction mixture was decanted by filtration and the remaining powder was soaked in $30.0 \mathrm{~mL}$ of THF at room temperature for 12 $\mathrm{h}$, after which the solvent was decanted and replaced with fresh deionized THF. Then, the solvent was switched to methanol and the process was repeated. Finally, the amorphous MOF nanosheets solid was collected by filtration and fully desolvated by heating under vacuum at $373 \mathrm{~K}$ for $24 \mathrm{~h}$.

Preparation of the MOF/Hexane Solution $(0.36 \mathrm{mg} / \mathrm{mL}) .30 .0 \mathrm{mg}$ of the fully desolvated amorphous MOF nanosheets were dispersed into $1.0 \mathrm{~L}$ of $n$-hexane and sonicated for $2 \mathrm{~h}$. The resultant mixture was centrifuged at 4,000 r.p.m for 20 minutes to remove the thicker amorphous MOF nanosheets and afford a transparent green solution $(800.0 \mathrm{~mL})$ The green solution was concentrated to $400.0 \mathrm{~mL}$ by rotate evaporation. To determine the concentration of the amorphous MOF nanosheets in hexane, $45.0 \mathrm{~mL}$ of the obtained solution was placed in a glass vial and dried in vacuum at $90{ }^{\circ} \mathrm{C}$ overnight. The concentration of the amorphous MOF nanosheets $(0.36 \mathrm{mg} / \mathrm{mL})$ was determined by comparing the weight changes of the glass vial. 
Fabrication of the PDMS\&MOF Gutter Layer. $0.2 \mathrm{~g}$ of $\mathrm{NH}_{2}-$ PDMS- $\mathrm{NH}_{2}$ (0.04 mmol, 1 equiv.) was dissolved in $10.0 \mathrm{~mL}$ of the green amorphous MOF nanosheet solution $\left(2.0 \% \mathrm{w} \mathrm{v}^{-1}\right.$, solution A). Another solution was prepared by dissolving $7.0 \mathrm{mg}$ of TMC $(0.0267$ $\mathrm{mmol})$ in $0.35 \mathrm{~mL}$ of pure $n$-hexane $\left(2.0 \% \mathrm{w} \mathrm{v} \mathrm{v}^{-1}\right.$, solution B). The two solutions were mixed for $2 \mathrm{~min}$ and $1.0 \mathrm{~mL}$ of the solution was then spin-coated $(1,000 \mathrm{rpm}, 10 \mathrm{~s})$ onto each PAN substrate $\left(19.63 \mathrm{~cm}^{2}\right.$, prewetted in deionized water for $60 \mathrm{~min}$ by sonication) to prepare PDMS\&MOF gutter layer. Then, $0.35 \mathrm{~mL}$ of TMC solution $(1.0 \% \mathrm{w}$ $\mathrm{v}^{-1}$, in $n$-hexane) was added into $10.0 \mathrm{~mL}$ of poly(DMS-co-BIBAPMS) solution $\left(2.0 \% \mathrm{w} \mathrm{v}^{-1}\right.$ in $n$-hexane). $1.0 \mathrm{~mL}$ of the mixture was spincoated $(1,000 \mathrm{rpm}, 10 \mathrm{~s})$ on the precoated PDMS\&MOF gutter layer to provide the PDMS\&MOF initiator layer. Finally, the precoated substrates were dried in vacuum for $24 \mathrm{~h}$ (1 mbar). Each of the obtained PDMS\&MOF membranes was tested the gas separation performance before coating the selective layer

Fabrication of the PEG Selective Layer. The CAP process on PDMS\&MOF coated PAN substrate was also conducted under ARGET-ATRP conditions. The PAN substrates with PDMS\&MOF initiator layer were immersed in an aqueous solution of $\mathrm{CuBr}_{2}(1 \mathrm{mM})$, Me 6 TREN $(3 \mathrm{mM})$, sodium ascorbate $(20 \mathrm{mM})$ and macrocross-linkers (PEGDMA, $200 \mathrm{mM}$ ). After the specified reaction time at room temperature, the substrates were removed, washed with DI water, soaked in water $(50.0 \mathrm{~mL})$ for $10 \mathrm{~min}$ and then dried in vacuo at $25^{\circ} \mathrm{C}$ for 24 $\mathrm{h}$ before the gas separation tests.

Simulation Study. We employ a computation model to simulate steady-state gas diffusion in our designed composite membranes, accounting for the effect of the amorphous MOF nanosheet inclusions within the PDMS substrate using a tensor resistance model. An axisymmetric domain $\left(r^{*}, z^{*}\right)$ is used to represent the diffusion around a single prototypical pore. Gas enters along the top of the membrane and leaves through the pore. The PAN substrate is impermeable.

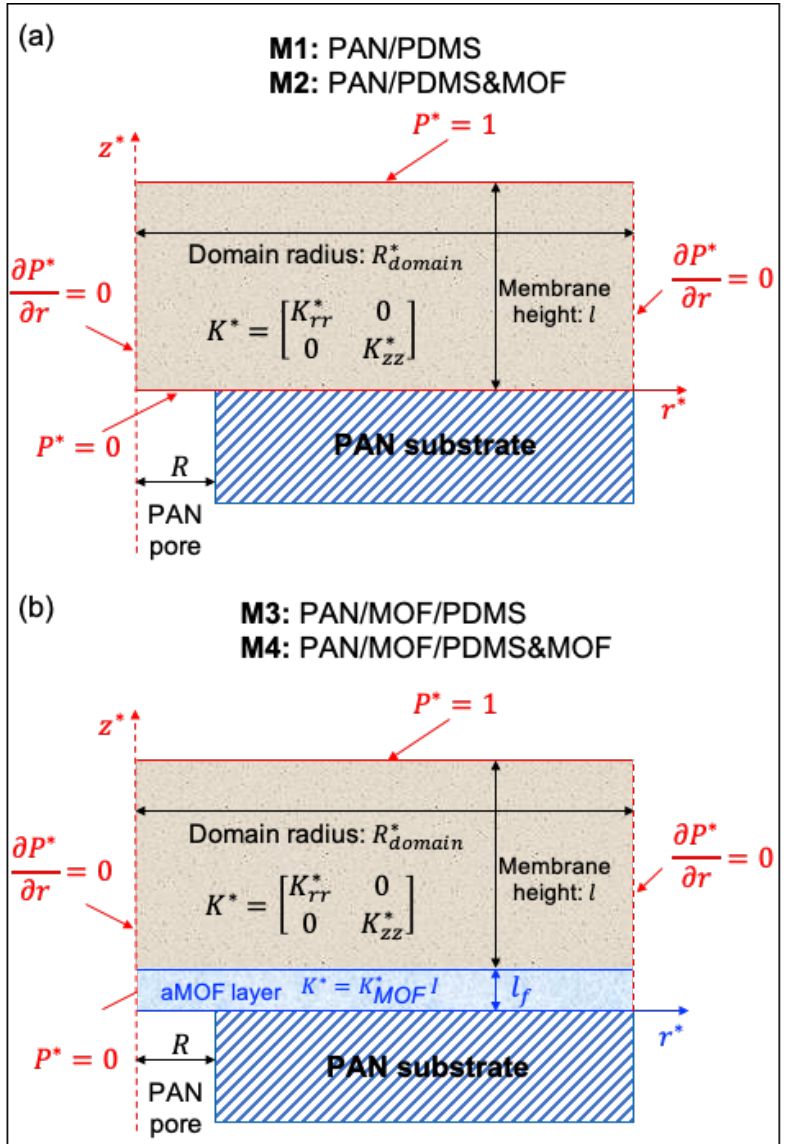

Scheme 1. Illustration of the membrane geometries and boundary conditions employed in this simulation study: (a) M1 and M2, (b) M3 and M4.
As shown in Scheme 1, for membrane configurations M1 and M2, the membrane consists of only the PDMS\&MOF composite (or plain PDMS), while for configurations M3 and M4 the intervening amorphous MOF nanosheet layer of higher permeability is also included. A finite volume method ${ }^{32}$ is used to solve the $\mathrm{CO}_{2}$ transport throughout the membrane via

$\boldsymbol{\nabla} \cdot r^{*} \boldsymbol{j}^{*}=0$

where the flux

$\boldsymbol{j}^{*}=-\boldsymbol{K}^{*} \cdot \boldsymbol{\nabla} P^{*}$

is given by the dot product of the tensor material permeability $K^{*}$ and gradient of $\mathrm{CO}_{2}$ partial pressure $P^{*}$ that would be in equilibrium with the local concentration. Variables are presented and solved for in nondimensionalized form (indicated by an asterisk), with length nondimensionalized by the pore radius $R$, permeability by the permeability of the bulk PDMS $K_{\mathrm{PDMS}}$, and pressure by the $\mathrm{CO}_{2}$ partial pressure $\Delta P$ applied over the membrane. The height of the composite membrane $l$ is as measured, and the radius of the domain $R_{\text {domain }}^{*}$ is related to the radius of the pore via the porosity of the PAN $\emptyset$ such that $R_{\text {domain }}^{*}=$ $1 / \emptyset$. The rate of $\mathrm{CO}_{2}$ transport through the membrane, per membrane surface area and applied pressure (the permeance) is calculated as

$$
Q=-\frac{K_{\mathrm{PDMS}} \emptyset}{\pi R} \int_{\text {pore }} \boldsymbol{j}^{*} \cdot \boldsymbol{k} d A^{*}
$$

where the integral is taken over the lower membrane surface at the top of the pore, and $\boldsymbol{k}$ is the unit vector in the axial direction.

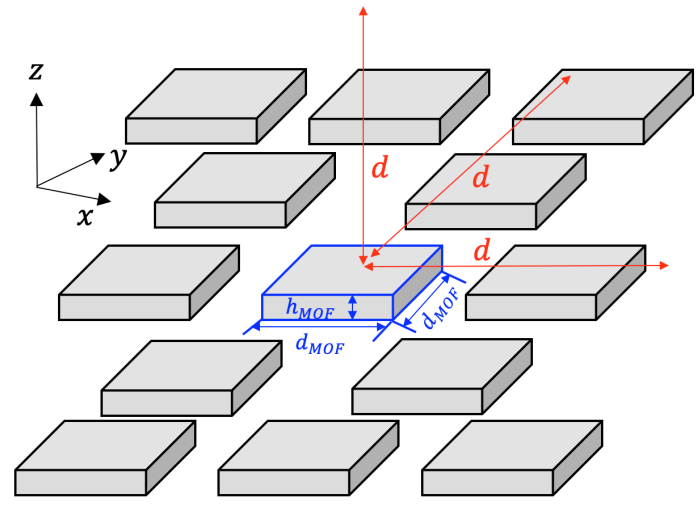

Scheme 2. Illustration of the diffusion model for the PDMS\&MOF materials consisting of a uniform array of varying aspect ratio amorphous MOF nanosheet inclusions.

The tensor permeability of the membrane composite $\boldsymbol{K}^{*}$ is calculated based on an inclusion model, as illustrated in Scheme 2. Rectangular cuboids of amorphous MOF nanosheets are spaced throughout the model PDMS composite material on a uniform square grid with spacing $d$. Each inclusion has a height (normal to the membrane) of $h_{\mathrm{MOF}}$, and square base dimension of $d_{\mathrm{MOF}}$. One dimensional resistance theory gives the permeability of the composite in the axial direction as

$$
K_{Z Z}^{*}=\left[1-\varphi^{\frac{1}{3}} \lambda^{\frac{2}{3}}+\frac{\varphi^{\frac{1}{3}} \lambda^{\frac{2}{3}}}{(\varphi / \lambda)^{\frac{2}{3}} K_{\mathrm{MOF}}^{*}+1-(\varphi / \lambda)^{\frac{2}{3}}}\right]^{-1}
$$

and in the radial direction (or any direction parallel to the membrane)

$$
K_{r r}^{*}=\left[1-(\varphi / \lambda)^{\frac{1}{3}}+\frac{\varphi^{\frac{1}{3}}}{(\varphi \lambda)^{\frac{2}{3}} K_{\mathrm{MOF}}^{*}+\lambda^{\frac{1}{3}}-(\varphi \lambda)^{\frac{2}{3}}}\right]^{-1}
$$


where $\varphi=h_{\mathrm{MOF}} d_{\mathrm{MOF}}^{2} / d^{3}$ is the volume fraction of amorphous MOF nanosheets in the PDMS composite material, $\varphi=h_{\mathrm{MOF}} / d_{\mathrm{MOF}}$ is the aspect ratio of the amorphous MOF nanosheet inclusions, and $K_{\mathrm{MOF}}^{*}$ is the nondimensional permeability of the amorphous MOF nanosheet material. Geometric considerations limit the inclusion aspect ratio to $\varphi^{-1 / 2}>\lambda>\varphi$. The $2 \mathrm{D}$ axisymmetric permeability tensor within the composite material hence becomes

$$
\boldsymbol{K}^{*}=\left[\begin{array}{cc}
K_{r r}^{*} & 0 \\
0 & K_{z z}^{*}
\end{array}\right]
$$

whereas within the intervening amorphous MOF nanosheet layer (for configurations M3 and M4) it is instead $\boldsymbol{K}^{*}=K_{\mathrm{MOF}}^{*} \boldsymbol{I}$, where $\boldsymbol{I}$ is the identity tensor.

Parameters used in the simulations were: The porosity and pore radius of the PAN substrate were measured by SEM and ImageJ software (porosity: $\sim 3.5 \%$ and average pore size: $\sim 11.5 \mathrm{~nm}$, Figure S15). The volume fraction of the amorphous MOF nanosheets in PDMS was around $1.0 \mathrm{v} / \mathrm{v} \%$, converted from the mass loading $(\sim 1.8 \%)$. The thickness $(\sim 230 \mathrm{~nm})$ of PDMS gutter layer was determined by SEM (Figure 2b). The $\mathrm{CO}_{2}$ permeability (294000 Barrer) of the amorphous MOF nanosheet was obtained from $\mathrm{MOF} /$ anodisc composite membranes fabricated by vacuum filtration. A sensitive study found the $\mathrm{CO}_{2}$ permeability of PDMS and the amorphous MOF nanosheet aspect ratio in PDMS are the main parameters that influence the simulated $\mathrm{CO}_{2}$ permeance.

\section{ASSOCIATED CONTENT}

\section{Supporting Information}

The Supporting Information is available free of charge on the ACS Publications website.

Materials, methods, characterization, SEM images, FTIR, XANES, XPS, TGA, ${ }^{1} \mathrm{H}$ NMR, $\mathrm{N}_{2}$ and $\mathrm{CO}_{2}$ adsorption isotherms, XRD, $\mathrm{CO}_{2} / \mathrm{N}_{2}$ separation performance, MOF dispersion and membrane images (PDF).

\section{AUTHOR INFORMATION}

\section{Corresponding Author}

*E-mail: daltonh@unimelb.edu.au

*E-mail: qiang.fu@uts.edu.au

*E-mail: paul.webley@monash.edu

*E-mail: gregghq@unimelb.edu.au

\section{Author Contributions}

D.H., Q.F., P.W., and G.Q. conceived the project. M.L. designed the experiments and wrote the manuscript. K.X., M.N., L.Z. and S.Z. contributed to the preparation and characterizations of materials. The manuscript was written with contributions from all authors. All authors have given approval to the final version of the manuscript.

\section{Notes}

The authors declare no competing financial interest.

\section{ACKNOWLEDGMENT}

The authors appreciate the Bio21 Advanced Microscopy Facility for the assistance with material characterization, Min Liu acknowledges the support from China Scholarship Council - University of Melbourne Research Scholarship (No. 201606260063). Qiang Fu acknowledges the Australian Research Council under the Future Fellowship (FT180100312).

\section{REFERENCES}

1. Baker, R. W.; Low, B. T. Gas separation membrane materials: A perspective. Macromolecules 2014, 47, 6999-7013.

2. Park, H. B.; Kamcev, J.; Robeson, L. M.; Elimelech, M.; Freeman, B. D. Maximizing the right stuff: The trade-off between membrane permeability and selectivity. Science $\mathbf{2 0 1 7}, 356$, eaab0530.

3. Galizia, M.; Chi, W. S.; Smith, Z. P.; Merkel, T. C.; Baker, R. W.; Freeman, B. D. 50th anniversary perspective: Polymers and mixed matrix membranes for gas and vapor separation: A review and prospective opportunities. Macromolecules 2017, 50, 7809-7843. 4. Merkel, T.; Bondar, V.; Nagai, K.; Freeman, B.; Pinnau, I. Gas sorption, diffusion, and permeation in poly (dimethylsiloxane). $J$. Polym. Sci., Part B: Polym. Phys. 2000, 38, 415-434.

5. Firpo, G.; Angeli, E.; Repetto, L.; Valbusa, U. Permeability thickness dependence of polydimethylsiloxane (PDMS) membranes. J. Membr. Sci. 2015, 481, 1-8.

6. Fu, Q.; Kim, J.; Gurr, P. A.; Scofield, J. M. P.; Kentish, S. E.; Qiao, G. G. A novel cross-linked nano-coating for carbon dioxide capture. Energ Environ. Sci. 2016, 9, 434-440.

7. Shishatskii, A.; Yampol'skii, Y. P.; Peinemann, K.-V. Effects of film thickness on density and gas permeation parameters of glassy polymers. J. Membr. Sci. 1996, 112, 275-285.

8. Yoo, M. J.; Kim, K. H.; Lee, J. H.; Kim, T. W.; Chung, C. W.; Cho, Y. H.; Park, H. B. Ultrathin gutter layer for high-performance thin-film composite membranes for $\mathrm{CO}_{2}$ separation. J. Membr. Sci. 2018, 566, 336-345.

9. Kattula, M.; Ponnuru, K.; Zhu, L.; Jia, W.; Lin, H.; Furlani, E. P. Designing ultrathin film composite membranes: The impact of a gutter layer. Sci. Rep. 2015, 5, 15016.

10. Wijmans, J.; Hao, P. Influence of the porous support on diffusion in composite membranes. J. Membr. Sci. 2015, 494, 78-85.

11. Shi, M.; Dong, C.; Wang, Z.; Tian, X.; Zhao, S.; Wang, J. Support surface pore structures matter: Effects of support surface pore structures on the $\mathrm{tfc}$ gas separation membrane performance over a wide pressure range. Chin. J. Chem. Eng. 2018, 27, 1807-1816. 12. Xie, K.; Fu, Q.; Xu, C.; Lu, H.; Zhao, Q.; Curtain, R.; Gu, D.; Webley, P. A.; Qiao, G. G. Continuous assembly of a polymer on a metal-organic framework (CAP on MOF): A $30 \mathrm{~nm}$ thick polymeric gas separation membrane. Energ Environ. Sci. 2018, 11, 544-550. 13. Liu, M.; Xie, K.; Nothling, M. D.; Gurr, P. A.; Tan, S. S. L.; Fu, Q.; Webley, P. A.; Qiao, G. G. Ultrathin metal-organic framework nanosheets as a gutter layer for flexible composite gas separation membranes. ACS Nano 2018, 12, 11591-11599.

14. Chen, H. Z.; Thong, Z.; Li, P.; Chung, T.-S. High performance composite hollow fiber membranes for $\mathrm{CO}_{2} / \mathrm{H}_{2}$ and $\mathrm{CO}_{2} / \mathrm{N}_{2}$ separation. Int. J. Hydrogen Energ. 2014, 39, 5043-5053. 15. Yampolskii, Y. Polymeric gas separation membranes. Macromolecules 2012, 45, 3298-3311.

16. Yaghi, O. M.; O'keeffe, M.; Ockwig, N. W.; Chae, H. K.; Eddaoudi, M.; Kim, J. Reticular synthesis and the design of new materials. Nature 2003, 423, 705.

17. Férey, G. Hybrid porous solids: Past, present, future. Chem. Soc. Rev. 2008, 37, 191-214.

18. Batten, S. R.; Champness, N. R.; Chen, X.-M.; Garcia-Martinez, J.; Kitagawa, S.; Öhrström, L.; O'Keeffe, M.; Suh, M. P.; Reedijk, J. Terminology of metal-organic frameworks and coordination polymers (IPUPAC recommendations 2013). Pur. Appl. Chem. 2013, $85,1715-1724$.

19. Bennett, T. D.; Cheetham, A. K. Amorphous metal-organic frameworks. Accounts Chem. Res. 2014, 47, 1555-1562.

20. Sanz, R.; Martinez, F.; Orcajo, G.; Wojtas, L.; Briones, D. Synthesis of a honeycomb-like Cu-based metal-organic framework and its carbon dioxide adsorption behaviour. Dalton T. 2013, 42, 2392-2398.

21. Ramohlola, K. E.; Monana, G. R.; Hato, M. J.; Modibane, K. D.; Molapo, K. M.; Masikini, M.; Mduli, S. B.; Iwuoha, E. I. Polyanilinemetal organic framework nanocomposite as an efficient electrocatalyst for hydrogen evolution reaction. Compos. Part B: Eng. 2018, 137, 129-139. 
22. Prestipino, C.; Regli, L.; Vitillo, J. G.; Bonino, F.; Damin, A.; Lamberti, C.; Zecchina, A.; Solari, P.; Kongshaug, K.; Bordiga, S. Local structure of framework $\mathrm{Cu}$ (II) in HKUST-1 metallorganic framework: Spectroscopic characterization upon activation and interaction with adsorbates. Chem. Mater. 2006, 18, 1337-1346. 23. Tan, K.; Chabal, Y. J., Interaction of small molecules within metal organic frameworks studied by in situ vibrational spectroscopy. In Metal-organic frameworks, IntechOpen: 2016; pp 19-36.

24. Huang, C.; Dong, J.; Sun, W.; Xue, Z.; Ma, J.; Zheng, L.; Liu, C.; Li, X.; Zhou, K.; Qiao, X.; Song, Q.; Ma, W.; Zhang, L.; Lin, Z.; Wang, T. Coordination mode engineering in stacked-nanosheet metal-organic frameworks to enhance catalytic reactivity and structural robustness. Nat. Commun. 2019, 10, 2779.

25. Dietzel, P. D.; Blom, R.; Fjellvåg, H. Base-induced formation of two magnesium metal-organic framework compounds with a bifunctional tetratopic ligand. Eur. J. Inorg. Chem. 2008, 2008, 36243632.

26. Dietzel, P. D.; Morita, Y.; Blom, R.; Fjellvåg, H. An in situ hightemperature single-crystal investigation of a dehydrated metalorganic framework compound and field-induced magnetization of one-dimensional metal-oxygen chains. Angew. Chem. Inter. Ed. 2005, 44, 6354-6358.

27. Dietzel, P. D.; Panella, B.; Hirscher, M.; Blom, R.; Fjellvåg, H. Hydrogen adsorption in a nickel based coordination polymer with open metal sites in the cylindrical cavities of the desolvated framework. Chem. Commun. 2006, 959-961.
28. Van Vleet, M. J.; Weng, T.; Li, X.; Schmidt, J. R. In situ, timeresolved, and mechanistic studies of metal-organic framework nucleation and growth. Chem. Rev. 2018, 118, 3681-3721.

29. Bachman, J. E.; Long, J. R. Plasticization-resistant $\mathrm{Ni}_{2}$ (dobdc)/polyimide composite membranes for the removal of $\mathrm{CO}_{2}$ from natural gas. Energ Environ. Sci. 2016, 9, 2031-2036.

30. Islam, M.; Buschatz, H. Assessment of thickness-dependent gas permeability of polymer membranes. Indian J. Chem. Techn. 2005, $12,88-92$.

31. Xie, K.; Fu, Q.; Webley, P. A.; Qiao, G. G. MOF scaffold for a high-performance mixed-matrix membrane. Angew. Chem. Inter. Ed. 2018, 57, 8597-8602.

32. Harvie, D. J. E. An implicit finite volume method for arbitrary transport equations. ANZIAM Journal 2010, 52, 1126-1145.

33. Merkel, T. C.; Lin, H.; Wei, X.; Baker, R. Power plant postcombustion carbon dioxide capture: An opportunity for membranes. J. Membr. Sci. 2010, 359, 126-139.

34. Wessling, M.; Lopez, M. L.; Strathmann, H. Accelerated plasticization of thin-film composite membranes used in gas separation. Sep. Purif. Technol. 2001, 24, 223-233.

35. D, D. M. Carbon dioxide capture: Prospects for new materials. Angew. Chem. Int. Ed. 2010, 49, 6058-6082. 
Insert Table of Contents artwork here

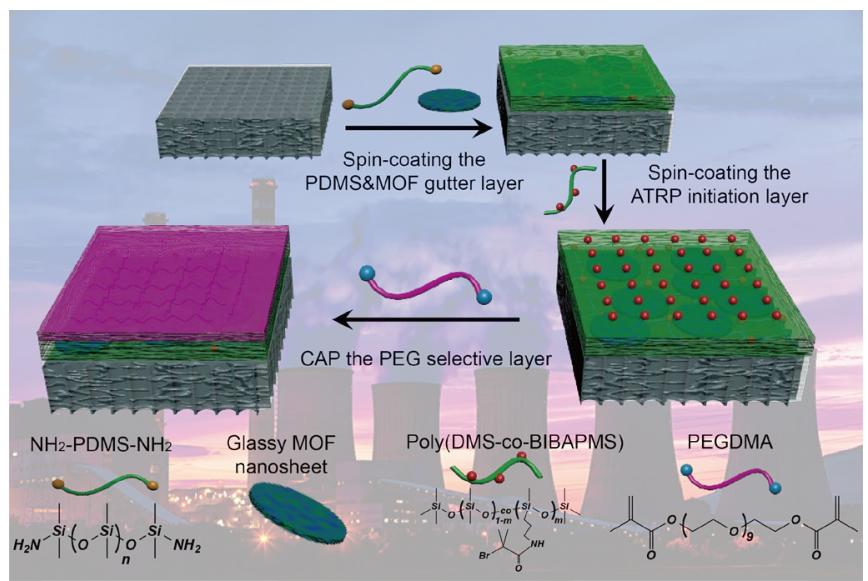

